\title{
EDITORIAL
}

\section{The European Respiratory Journal targets tuberculosis}

\author{
C. Lange*, W.W. Yew ${ }^{\#}$, G.B. Migliori ${ }^{\top}$ and M. Raviglione ${ }^{+}$
}

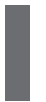
$\mathrm{n}$ the most recent report [1, 2], the World Health Organization (WHO) estimates that in 20089.4 million new tuberculosis (TB) cases occurred and that 1.8 million people died of TB. Tuberculosis remains a leading cause of morbidity and mortality in many areas of the world, ranking at the seventh position of all causes of deaths worldwide and being second only to AIDS as an infectious cause of death [3]. All regions of the world are affected, with the highest per capita rates in sub-Saharan Africa and the highest absolute numbers in Asia; however, the Middle East, Europe, especially Eastern Europe, and the Americas are also paying a heavy toll to the disease. It is very likely that the number of individuals who are latently infected with Mycobacterium tuberculosis, estimated to be about a third of humanity, has never been higher during human evolution than today.

In the past decades, huge efforts have been made and ambitious goals have been set, such as halving prevalence and mortality by 2015 compared to 1990 and eliminating TB by 2050 [4], to combat TB at different levels: by governments, multilateral organisations (e.g. WHO, the World Bank), new financing mechanisms (e.g. the Global Fund, UNITAID), non-governmental organisations, foundations (e.g. The Bill \& Melinda Gates Foundation, the Rockefeller Foundation), civil society, the corporate sector and professional societies (e.g. the American Thoracic Society and the European Respiratory Society (ERS)). As a result of these efforts, nearly 6 million lives have been saved compared to the standards of care before 1995 and 36 million people have been cured since 1995. Furthermore, the Millennium Development Goal-related TB target of halting the increase and beginning to make incidence decline, seems to have been reached in 2004, although the decline is extremely slow at this point. However, a more rapid decline in incidence and other international goals, particularly that of elimination by 2050, will be hard, if not impossible, to reach with current tools [2, 4].

Leaving aside the funding constraints, the most important reasons impeding rapid decline in incidence and elimination, are related to late and low case detection, M. tuberculosis/HIV co-infection and anti-TB drug resistance.

There are often delays in detection of people with TB, linked to poor health service access and system problems both in public and private clinics. For instance, private for-profit practitioners are rarely engaged by national programmes, detect too late and treat often without following international recommendations

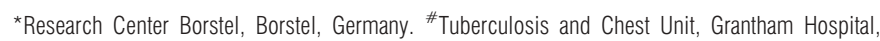
Hong Kong, China. "World Health Organization Collaborating Centre for TB and Lung Disease, Fondazione S. Maugeri, Tradate, Italy. ${ }^{+}$World Health Organization, Geneva, Switzerland.

CORRESPONDENCE: C. Lange, Division of Clinical Infectious Diseases, Medical Clinic, Research Center Borstel, Parkallee 35, 23845 Borstel, Germany. E-mail: clange@fz-borstel.de
[5]. This translates into uninterrupted transmission within the community and perpetuation of the infection. A response must be based on the identification of the most vulnerable groups, such as contacts, people living with HIV (PLHIV), diabetics and the poorest, and on the pursuit of early case detection and chemoprophylaxis for those not yet sick.

Vulnerability of individuals with CD4+ T-cell related immunodeficiencies has caused an enormous rise in the number of cases of TB in areas where HIV infection is highly endemic, especially in sub-Saharan Africa [1, 2]. The rapid rise of HIV infection in some Eastern European settings is presently an issue of major concern $[1,2]$.

Emergence of anti-TB multidrug resistance (MDR-TB) is now a very serious threat to the gains in TB control, especially in the former Soviet Union countries, China and India [6]. Antibiotic drug resistance in $M$. tuberculosis is of major concern as no new drug has been developed and licensed for the treatment of TB for more than $40 \mathrm{yrs}$. In particular, the increasing prevalence of isoniazid drug resistance will weaken the effectiveness and acceptance of TB chemoprophylaxis efforts.

In principle, improvements in TB control and efforts towards elimination require advances in three areas: prevention, diagnosis and treatment.

Primary prevention of TB by vaccination with Mycobacterium bovis bacille Calmette-Guerin (BCG) is a tool with major limitations, although BCG has been the mostly used vaccine worldwide. Whereas BCG vaccination is effective to reduce the severity of TB in children, it has little and unpredictable effects among adults in reducing the most infectious forms of TB. Thus, its impact on interrupting transmission is negligible. Vaccines that have a substantial impact on TB prevention are urgently needed to reduce individual suffering and incidence worldwide.

Secondary prevention against $\mathrm{TB}$, e.g. treatment of latent $\mathrm{TB}$ infection by isoniazid preventive therapy (IPT) for high-risk groups, including PLHIV or contacts with a positive tuberculin skin test or a positive interferon- $\gamma$ release assay (IGRA) result, has an efficacy of up to $70 \%$ if the therapy is continued for $\geqslant 6$ 9 months [7]. However, large-scale feasibility and acceptance of IPT are highly variable in industrialised countries and endemic countries alike.

So far, most of the advances in the field of TB over the past decade have been achieved in the area of diagnosis [8]. The immunodiagnosis of individuals at risk for the development of TB has been added to our armamentarium, although its usefulness in comparison with the tuberculin skin test is not entirely clear. IGRAs have now become widely available in low-incidence countries for the detection of latent TB infection. 
Although conclusive evidence is still lacking, especially in vulnerable populations, the positive predictive value of IGRAs for the development of TB in a latently infected person may one day be of relevance for decisions on early treatment [9]. However, as in the case of tuberculin skin testing, current generations of IGRAs are not able to distinguish individuals with active or past TB from those with latent TB infection and are, therefore, of limited value at the moment [10].

Important advances have also been made in the field of the rapid molecular diagnosis of tuberculosis. Line probe assays used for the amplification of specific DNA sequences harbouring genetic mutations related to $M$. tuberculosis drug resistance have a sensitivity and specificity similar to that of modern M. tuberculosis liquid culture systems and drug susceptibility testing [11]. Thus, they allow for more rapid diagnosis of $M$. tuberculosis drug resistance (including MDR-TB and extensively drug resistant (XDR)-TB from acid-fast bacilli smear-positive specimens [12]).

The development of new, potent and tolerable drugs against M. tuberculosis has been neglected for a long time. Although some antibiotics like the fluoroquinolones (especially moxifloxacin and gatifloxacin) and linezolid, developed for other infectious diseases, have in vitro and in vivo activity against M. tuberculosis, novel drugs without cross-resistance against existing antituberculosis drugs are urgently needed. Few candidate drugs (including moxifloxacin) have entered phase II clinical trials [13, 14]; however, it is likely that novel drugs could be available for the treatment of patients with MDR- and XDR-TB in the next 3-5 yrs. However, of crucial importance will be the testing of combination regimens with novel compounds if one has to have a regimen that will be usable for all TB patients in settings where MDR-TB is highly prevalent. In addition, regimens that could shorten current 6-month therapies are desirable to facilitate adherence, and shorter chemoprophylaxis regimens are also necessary if treatment of latent infection has to be widened to large-scale intervention among populations or individuals at risk.

The European Respiratory Journal (ERJ) has contributed substantially to the dissemination of advances in knowledge regarding $\mathrm{TB}$ in the past and is committed to doing so in the future [15]. In the forthcoming months, the ERJ will issue a review series on TB covering, in depth, the clinical management of patients with TB and individuals suspected to be latently infected with $M$. tuberculosis. These most important topics will include updated guidelines for TB contact tracing in Europe, recommendations for the management of patients with tumour necrosis factor antagonist therapies who are at risk of $\mathrm{TB}$, treatment updates of active $\mathrm{TB}$ and latent $\mathrm{TB}$ infection, and state-of-the-art management of individuals with TB and HIV co-infection, as well as the relationship of noncommunicable diseases with TB. The first article in this series appears in the current issue of the ERJ [16].

Improvements in the care of patients with $\mathrm{TB}$ and global TB control will require concerted action from many stakeholders. As a partner in this process, the ERS supports TB research within the TB-PAN-NET, a large consortium for research on MDR-TB funded by the European Commission and has joined forces with the Tuberculosis Network European Trials Group (TBNET) in a Clinical Research Cooperation (CRC). As the most important publication organ of the ERS, the ERJ will strengthen this process by continuing to disseminate clinically important advances in the field of TB.

\section{STATEMENT OF INTEREST}

None declared.

\section{REFERENCES}

1 World Health Organization. Global tuberculosis control: surveillance, planning, financing. WHO Report 2009. World Health Organization Document: WHO/HTM/TM2009.4111-303.

2 World Health Organization. Global tuberculosis control: a short update to the 2009 report. World Health Organization Document: WHO/HTM/TB2009.4261-48.

3 World Health Organization. Fact Sheet: Top ten causes of deaths. World Health Organization Document, 2008. www.who.int/ mediacentre/factsheets/fs310/en/index.html Date last updated: October 2008. Date last accessed: April 2010.

4 Dye C, Maher D, Weil D, et al. Targets for global tuberculosis control. Int J Tuberc Lung Dis 2006; 10: 460-462.

5 Uplekar M, Pathania V, Raviglione M. Private practitioners, public health, weak links in tuberculosis control. Lancet 2001; 358: 912-916.

6 World Health Organization. Multidrug and extensively drugresistant TB (M/XDR-TB). 2010 Global report on surveillance and response. WHO, Geneva, 2010. World Health Organization Document WHO/HTM/TB2010.31-71.

7 Akolo C, Adetifa I, Shepperd S, et al. Treatment of latent tuberculosis infection in HIV infected persons. Cochrane Database Syst Rev 2010; 1: CD000171.

8 Lange C, Mori T. Advances in the diagnosis of tuberculosis, Respirology 2010; 15: 220-240.

9 Diel R, Loddenkemper R, Meywald-Walter K, et al. Predictive value of a whole blood IFN-gamma assay for the development of active tuberculosis disease after recent infection with Mycobacterium tuberculosis. Am J Respir Crit Care Med 2008; 177: 1164-1170.

10 Lange C, Pai M, Drobniewski F, et al. Interferon-gamma release assays for the diagnosis of active tuberculosis sensible or silly? Eur Respir J 2009; 33: 1250-1253.

11 Hillemann D, Rusch-Gerdes S, Richter E. Evaluation of the GenoType(R) MTBDRplus. Assay for rifampicin, isoniazid susceptibility testing of Mycobacterium tuberculosis strains and in clinical specimens. J Clin Microbiol 2007; 45: 2635-2640.

12 Hillemann D, Rusch-Gerdes S, Richter E. Feasibility of the GenoType MTBDRsl assay for fluoroquinoloneamikacin-capreomycin, and ethambutol resistance testing of Mycobacterium tuberculosis strains and clinical specimens. J Clin Microbiol 2009; 47: 1767-1772.

13 Dorman SE, Johnson JL, Goldberg S, et al. Substitution of moxifloxacin for isoniazid during intensive phase treatment or pulmonary tuberculosis. Am J Respir Crit Care Med 2009; 180: 273-280.

14 Diacon AH, Pym A, Grobusch $\mathrm{M}$, et al. The diarylquinoline TMC207 for multidrug-resistant tuberculosis. N Engl J Med 2009; 360: 2397-2405.

15 Migliori GB, Loddenkemper R, Blasi F, et al. 125 years after Robert Koch's discovery of the tubercle bacillus: the new XDR-TB threat. Is "science" enough to tackle the epidemic? Eur Respir J 2007; 29: 423-427.

16 Erkens CGM, Kamphorst M, Abubakar I, et al. Tuberculosis contact investigation in low prevalence countries: a European consensus. Eur Respir J 2010; 36: 925-949. 